\title{
Development of a Generalized Theoretical Model for the Response of a Phase/Doppler Measurement System to Arbitrarily Oriented Fibers Illuminated by Gaussian Beams
}

\author{
Scott A. Schaub \\ James A. Lock \\ Cleveland State University, j.lock@csuohio.edu \\ Follow this and additional works at: https://engagedscholarship.csuohio.edu/sciphysics_facpub \\ nmir A. Naqwi \\ Part of the Physics Commons
}

How does access to this work benefit you? Let us know!

\section{Publisher's Statement}

This paper was published in Applied Optics and is made available as an electronic reprint with the permission of OSA. The paper can be found at the following URL on the OSA website: http://www.opticsinfobase.org/ao/abstract.cfm?URI=ao-37-33-7842. Systematic or multiple reproduction or distribution to multiple locations via electronic or other means is prohibited and is subject to penalties under law.

\section{Original Citation}

Schaub, Scott A., James A. Lock, and Amir A. Naqwi. "Development of a Generalized Theoretical Model for the Response of a Phase/Doppler Measurement System to Arbitrarily Oriented Fibers Illuminated by Gaussian Beams." Applied Optics 37 (1998): 7842-7855.

\section{Repository Citation}

Schaub, Scott A.; Lock, James A.; and Naqwi, Amir A., "Development of a Generalized Theoretical Model for the Response of a Phase/Doppler Measurement System to Arbitrarily Oriented Fibers Illuminated by Gaussian Beams" (1998). Physics Faculty Publications. 96.

https://engagedscholarship.csuohio.edu/sciphysics_facpub/96

This Article is brought to you for free and open access by the Physics Department at EngagedScholarship@CSU. It has been accepted for inclusion in Physics Faculty Publications by an authorized administrator of EngagedScholarship@CSU. For more information, please contact library.es@csuohio.edu. 


\title{
Development of a generalized theoretical model for the response of a phase/Doppler measurement system to arbitrarily oriented fibers illuminated by Gaussian beams
}

\author{
Scott A. Schaub, James A. Lock, and Amir A. Naqwi
}

\begin{abstract}
We present a generalized theoretical model for the response of the phase/Doppler (P/D) measurement system to light scattered by cylindrical fibers. This theoretical model is valid for arbitrary fiber diameters and refractive indices, for Gaussian incident beams, and it accounts for arbitrary fiber orientations, fiber positions, and effects that are due to the two-dimensional receivers. The generalized P/D computer model (GPDCM) is the extension of an earlier study by the authors, combining past P/D simulation methodology with recent developments in modeling light scattering by tilted cylindrical fibers. A FORTRAN computer program that implements the GPDCM theoretical development was written and tested against known P/D results and physical expectations. To illustrate the capabilities of the GPDCM, we present computation results, comparing the effect of fiber tilt, fiber position, and receiver aperture on the performance of $\mathrm{P} / \mathrm{D}$ systems configured in backscatter and sidescatter arrangements. Calculations show that the effects of fiber tilt and position are most pronounced in the backscatter P/D arrangement, resulting in broadening of the measured phase distribution. The calculated mean phase shifts, however, were found to be essentially independent of the above factors. Computational results also showed that the effect of fiber tilt and position on phase-distribution measurements can be reduced through proper choice of aperture shape and by imposition of threshold criteria on measurable signal characteristics such as the amplitude ratio and visibilities. The GPDCM provides a computational tool that will be valuable in the design, optimization, and evaluation of P/D fiber measurement systems. (C) 1998 Optical Society of America

OCIS codes: $\quad 290.0290,280.1100,120.3180$.
\end{abstract}

\section{Introduction and Background}

Instrumentation that would provide real-time, online determination of the diameter distribution of small fibers has important applications in several fiber-based industries, including those producing insulations, reinforcements, and optical fibers. These fibers are produced from many different materials, including a variety of glasses as well as synthetic materials such as polyethylene and polypropylene. Reliable, on-line instrumentation would provide fiber industries a valuable tool to

S. A. Schaub is with Mission Research Corporation, 1720 Randolph Road, S.E., Albuquerque, New Mexico 87106. J. A. Lock is with the Department of Physics, Cleveland State University, Cleveland, Ohio 44115. A. A. Naqwi is with TSI, Incorporated, 500 Cardigan Road, St. Paul, Minnesota 55126. The email address for S. A. Schaub is sschaub@ricochet.net.

Received 7 April 1998; revised manuscript received 31 August 1998.

0003-6935/98/337842-14\$15.00/0

(C) 1998 Optical Society of America assist in fiberizing research and development, quality-control monitoring, and potentially to improve plant production efficiencies by means of online monitoring and feedback. Although there is currently no accepted method for performing online fiber-diameter measurements, one technique showing some promise, which we examine in this work, is based on phase/Doppler (P/D) laser light scattering.

For many years $\mathrm{P} / \mathrm{D}$ measurement instrumentation has been used successfully for sizing dynamic sprays and spherical aerosols. Many studies have been presented in the literature that have addressed design considerations as well as the applicability of the $\mathrm{P} / \mathrm{D}$ technique to spherical aerosol sizing under various measurement conditions. ${ }^{1-5}$ The $\mathrm{P} / \mathrm{D}$ method has proved attractive for in situ measurements, as it offers several important advantages over alternative techniques, including nonintrusive operation, high spatial resolution, simultaneous particle size and velocity measurements, and measurements that are independent of absolute intensity. Despite 
the successes in spherical aerosol sizing, only recently has serious effort been made to generalize the $\mathrm{P} / \mathrm{D}$ measurement technique to sizing cylindrical fibers.

One complication in sizing fibers arises from the fact that, for many applications, the fibers are presented to the measurement volume in arbitrary orientations. This fiber tilt effect has no analogy in spherical aerosol sizing and results in additional uncertainty in measurement of the $\mathrm{P} / \mathrm{D}$ phase shift and thus fiber diameter. Another difference is that the fibers scatter light as a thin cone (or thin sheet in the case of normal beam/fiber incidence), thus resulting in a reduced region of space in which to position the instrumentation. The successful design of a $\mathrm{P} / \mathrm{D}$ system for sizing cylindrical aerosols will require us to address several specific design objectives. Two of the most important include:

1. Identification of experimental configurations that provide a one-to-one (preferably linear) relationship between the $\mathrm{P} / \mathrm{D}$ phase shift and fiber diameter. This aspect of the $\mathrm{P} / \mathrm{D}$ instrument development was examined in an earlier study with a plane-wave, perpendicular fiber model. ${ }^{6}$

2. Identification of experimental configurations and/or signal-processing options that successfully manage the problems associated with fiber tilt. Fiber tilt, translation, and finite receiver effects are all examined with the $\mathrm{P} / \mathrm{D}$ model developed in this paper.

To complicate matters further, the above design goals must be accomplished within the overall restrictions established by various physical and operational constraints imposed by the particular application under consideration. In many cases, such as in the sizing of fiberglass insulation, these restrictions can limit the choice of scattering angles and $\mathrm{P} / \mathrm{D}$ lens focal lengths that can be used effectively. ${ }^{6}$

Although the effects of fiber tilt can and must be evaluated experimentally, there are several important reasons why computer modeling represents a valuable component in $\mathrm{P} / \mathrm{D}$ instrument design.

1. When experimental testing is performed, only a limited number of experimental configurations can be examined, because of limited available hardware. Computer modeling allows investigation of any possible configuration prior to fabricating actual hardware components, thus limiting the expense associated with system design and optimization.

2. Also, experimental measurements tend to be somewhat time-consuming. The ability to predict the response of various experimental configurations by means of computer models allows a more rapid investigation of the relevant parameter space than could be accomplished with experimental measurements alone.

3. Although very fine fibers exist in many fiberglass products, one cannot, in general, produce fibers smaller than $\sim 1.5 \mu \mathrm{m}$ and still maintain the unifor- mity that is necessary for experimental testing of a single fiber. As such, computer modeling might represent the only practical means for predicting the response of the $\mathrm{P} / \mathrm{D}$ instrument to fibers smaller than $\sim 1.5 \mu \mathrm{m}$ in diameter. In addition, computer modeling allows for evaluation of the system response for diameters that might not be available in the set of fiber test samples.

As is generally the case, however, to obtain the most complete understanding of the problem at hand, theory and experiment should be pursued in parallel as part of a comprehensive research program. Input from each approach provides unique insight into the underlying fundamental physics that cannot always be easily determined with the complementary approach.

In earlier modeling efforts, Mignon et al.,${ }^{7}$ Naqwi and Jensen, ${ }^{8}$ and Naqwi et al. ${ }^{9}$ considered modeling light scattering by tilted fibers in a P/D system from a geometrical-optics viewpoint and considered only the contribution of light transmitted directly through a dielectric fiber. Schaub et al. ${ }^{6}$ performed modeling of finite absorbing cylindrical fibers in a P/D system, using a Lorenz-Mie analogy. Using this method, we examined the linearity of the phase-diameter relationship for the fiberglass measurement application but assumed plane-wave incident fields and perpendicular fiber orientations.

In this paper we address some of the limitations of earlier modeling and present a generalized $\mathrm{P} / \mathrm{D}$ computer model (GPDCM) for the response of the P/D system to cylindrical fibers that includes the ability to account for arbitrarily oriented fibers, focused Gaussian incident beams, and effects that are due to the finite nature of the receiver assembly. The capabilities provided by this higher-fidelity model of the P/D system are important in obtaining more realistic insight into instrument performance.

In Section 2 we present a discussion of the development of the GPDCM, including an outline of the relevant geometries and coordinate transformations required, a presentation of the light-scattering formalism, a brief discussion on modeling of the receiver assembly, and discussion of the output quantities of interest. In Section 3 we outline some general observations regarding the light scattering that can be made from a purely analytical viewpoint. In Section 4 we illustrate the computational capabilities of the GPDCM model by examining the effect of fiber tilt and fiber translation on $\mathrm{P} / \mathrm{D}$ instrument performance. Finally, in Section 5 we summarize the major findings that affect the design of $\mathrm{P} / \mathrm{D}$ fiber sizing instrumentation.

\section{Theoretical Modeling}

\section{A. Development of the Generalized Phase/Doppler Computer Model}

The calculation of electromagnetic scattering of the crossed laser beams of a $\mathrm{P} / \mathrm{D}$ particle sizing instrument by a tilted fiber is complicated by the mismatch 

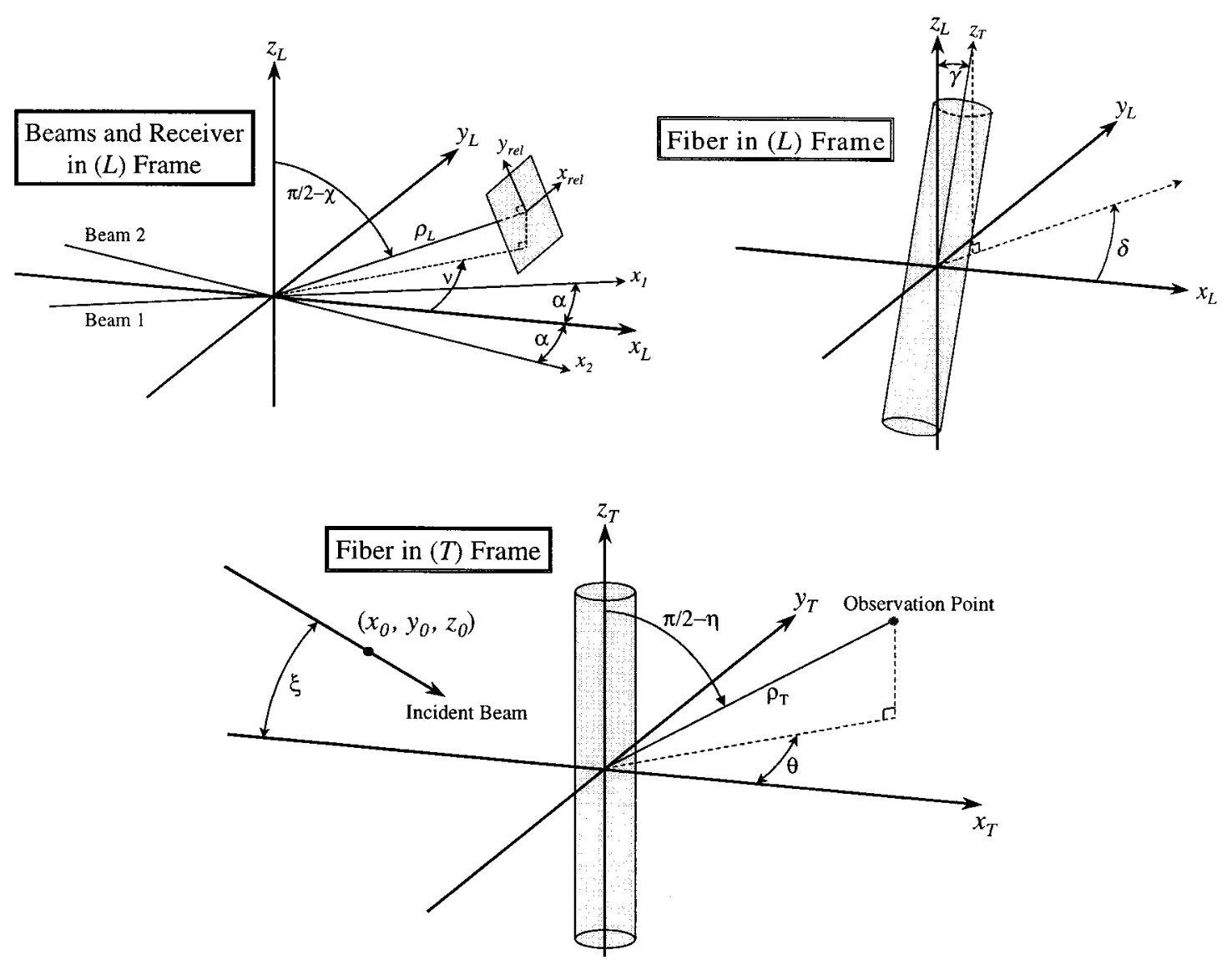

Fig. 1. Schematic of laboratory $(L)$ and theory $(T)$ coordinate systems used in theoretical modeling.

between the laboratory specification of the input parameters and the specific geometry employed in the numerical computation of the scattered fields. In the laboratory reference frame (denoted by subscript $L$ ), the centers of the focal waists of the two crossed laser beams coincide at the origin of the $x_{L}, y_{L}, z_{L}$ coordinate system and their propagation directions lie in the $x_{L}-y_{L}$ plane (see Fig. 1). Incident-beam crossover angles $\pm \alpha$ are measured relative to the $x_{L}$ axis and are determined by the beam separation at the transmitting lens and the transmitter focal length. The two incident Gaussian beams have their electric-field polarizations in either the direction of the $z_{L}$ axis (denoted as parallel or $m$ polarization) or in the $x_{L}-y_{L}$ plane (denoted as perpendicular or $e$ polarization). The fiber is both displaced from and tilted with respect to the $z_{L}$ axis. In the theory reference frame (denoted by the subscript $T$ ), however, the fiber's symmetry axis coincides with the $z_{T}$ axis of the $x_{T}, y_{T}, z_{T}$ coordinate system, and the propagation direction of an incident beam lies in the $x_{T}-z_{T}$ plane.

To compute scattering of the crossed incident beams by the fiber, the input parameters of the beams, fiber, and detectors must first be converted from the $(L)$ frame to the $(T)$ frame. The scattered fields produced by each of the two incident beams and evaluated at a point on the $\mathrm{P} / \mathrm{D}$ receiver aperture are then computed in the $(T)$ frame. The scattered fields are then converted from the $(T)$ frame back to the $(L)$ frame, where the respective components of the two sets of scattered fields are added together. Finally, the total scattered Poynting vector is calculated and integrated over the receiver aperture to produce the total light signal incident on the $\mathrm{P} / \mathrm{D}$ receiver assembly.

In the following development the subscripts $L, L^{\prime}$, or $T$ denote whether the quantity under consideration is specified in the laboratory frame, the translated laboratory frame, or the theory frame, respectively. The subscript $j=1,2$ denotes the first or second $\mathrm{P} / \mathrm{D}$ incident beam or the scattered wave produced by it. The superscript inc or scatt denotes whether the quantity under consideration pertains to a field incident on the fiber or scattered by it, respectively.

\section{Specification of the Input Parameters in the} Laboratory Reference Frame

The unit vector in the propagation direction of the $j$ th incident beam in the $(L)$ frame is

$$
\hat{\mathbf{k}}_{j, L}^{\mathrm{inc}}=\cos \alpha \hat{\mathbf{u}}_{x L} \pm \sin \alpha \hat{\mathbf{u}}_{y L},
$$

where the upper sign corresponds to the $j=1$ beam, the lower sign corresponds to the $j=2$ beam, and the quantity $\hat{\mathbf{u}}$ denotes a unit vector. The wavelength of each beam is $\lambda$ with a corresponding wave number 
$k=2 \pi / \lambda$. The focal plane of each incident beam is assumed to have a Gaussian amplitude profile with a $1 / e$ electric field half-width $w_{0}$. The center of the focal waist of each beam is at the origin of the $(L)$ frame.

The fiber is assumed to be a homogeneous infinite circular cylinder of radius $a$ with a complex refractive index $\bar{n}$. The fiber symmetry axis crosses the $x_{L}-y_{L}$ plane at $\left(x_{\mathrm{fib}}, y_{\mathrm{fib}}, 0\right)$, and it is tilted with respect to the $z_{L}$ axis by the spherical coordinate system polar angle $\gamma$ and azimuthal angle $\delta$ (see Fig. 1). The center of the $P / D$ receiver aperture is a distance $\rho_{L}$ from the laboratory origin and is in the direction of the spherical coordinate system polar angle $\pi / 2-\chi$ and azimuthal angle $\nu$. The receiver plane is oriented normal to the radial direction, and the coordinates of a point on the receiver aperture with respect to the center position are $\left(x_{\text {rel }}, y_{\text {rel }}\right)$, where the $x_{\text {rel }}$ axis is parallel to the $x_{L}-y_{L}$ plane. A point on the receiver aperture (superscript $d$ ) is then given in the $(L)$ frame by

$$
\begin{aligned}
& x_{L}^{d}=\rho_{L} \cos \chi \cos v-x_{\text {rel }} \sin v-y_{\text {rel }} \sin \chi \cos v, \\
& y_{L}^{d}=\rho_{L} \cos \chi \sin v+x_{\text {rel }} \cos v-y_{\text {rel }} \sin \chi \sin v, \\
& z_{L}^{d}=\rho_{L} \sin \chi+y_{\text {rel }} \cos \chi .
\end{aligned}
$$

\section{Specification of the Input Parameters in the Theory Frame}

In the theory frame $(T)$, the unit vector in the propagation direction of an incident beam is

$$
\hat{\mathbf{k}}_{j, T}^{\text {inc }}=\cos \xi \hat{\mathbf{u}}_{x T}-\sin \xi \hat{\mathbf{u}}_{z T}
$$

and the center of the beam focal waist is located at the point $\left(x_{0}, y_{0}, z_{0}\right)$. The incident beam is said to be $\epsilon$ polarized if its electric field is in the positive $y_{T}$ direction as in Ref. 10, and it is $\mu$ polarized if its magnetic field is in the negative $y_{T}$ direction. Evidently, the theory frame for the $j=1$ beam differs from the theory frame of the $j=2$ beam. Also, note that the $\epsilon$ and $\mu$ polarizations employed in the $(T)$ frame are not identical with the $e$ and $m$ polarizations specified in the $(L)$ frame. The center of the receiver aperture is a distance $\rho_{T}$ from the origin of the $(T)$ frame. An observation point on the receiver aperture is in the direction of the spherical coordinate system polar angle $\pi / 2-\eta$ and azimuthal angle $\theta$. The angles $\theta$ and $\eta$ are known as the horizontal and vertical scattering angles, respectively. The scattered wave at the receiver is $\epsilon$ polarized if its electric field lies in the $x_{T}-y_{T}$ plane as in Ref. 10, and it is $\mu$ polarized if its magnetic field lies in the $x_{T}-y_{T}$ plane.

\section{Transformation of Quantities from the Laboratory Frame to the Theory Frame}

A translation of the scattering apparatus in the $x_{L}$ direction by the distance $-x_{\text {fib }}$ and in the $y_{L}$ direction by the distance $-y_{\text {fib }}$ moves the origin of coordinates from the beam intersection point to a point on the fiber axis. The beam intersection point then has the coordinates $\left(-x_{\mathrm{fib}},-y_{\mathrm{fib}}, 0\right)$ in the translated labora- tory frame $\left(L^{\prime}\right)$, and the location of a point on the receiver is given by

$$
\begin{aligned}
& x_{L^{\prime}}^{d}=x_{L}^{d}-x_{\mathrm{fib}}, \\
& y_{L^{\prime}}^{d}=y_{L}^{d}-y_{\mathrm{fib}}, \\
& z_{L^{\prime}}^{d}=z_{L^{d}}^{d} .
\end{aligned}
$$

The distance from the origin of the $\left(L^{\prime}\right)$ frame to the point on the receiver aperture is

$$
\rho_{L^{\prime}}=\left[\left(x_{L^{\prime}}^{d}\right)^{2}+\left(y_{L^{\prime}}^{d}\right)^{2}+\left(z_{L^{\prime}}^{d}\right)^{2}\right]^{1 / 2},
$$

and its spherical coordinate system polar and azimuthal angles are $\chi^{\prime}$ and $\nu^{\prime}$, respectively, where

$$
\begin{aligned}
\tan \chi^{\prime} & =\frac{z_{L^{\prime}}^{d}}{\left[\left(x_{L^{\prime}}^{d}\right)^{2}+\left(y_{L^{\prime}}^{d}\right)^{2}\right]^{1 / 2}}, \\
\tan \nu^{\prime} & =\frac{y_{L^{\prime}}^{d}}{x_{L^{\prime}}^{d}} .
\end{aligned}
$$

A rotation of

$$
\left[R_{j}\right]=\left[R_{z L}(-\phi)\right]\left[R_{y L}(-\gamma)\right]\left[R_{z L}(-\delta)\right]
$$

connects the translated laboratory frame $\left(L^{\prime}\right)$ with the theory frame $(T)$, where the angle $\phi$ is defined by

$$
\begin{aligned}
& \cos \phi=\cos \beta \cos \gamma /\left(\cos ^{2} \beta \cos ^{2} \gamma+\sin ^{2} \beta\right)^{1 / 2}, \\
& \sin \phi=-\sin \beta /\left(\cos ^{2} \beta \cos ^{2} \gamma+\sin ^{2} \beta\right)^{1 / 2},
\end{aligned}
$$

with

$$
\beta=\delta \mp \alpha .
$$

The upper sign in Eq. (15) corresponds to the $j=1$ beam, the lower sign corresponds to the $j=2$ beam, and the notation $\left[\mathrm{R}_{z L}(-\delta)\right]$, for example, denotes a rotation of the light-scattering apparatus by the angle $-\delta$ about the $z_{L}$ axis. The first two rotations of Eq. (12) align the fiber axis with the $z_{T}$ axis, and the final rotation places the propagation direction of the incident beam in the $x_{T}-z_{T}$ plane.

The rotation $\left[R_{j}\right]$ converts the unit vector in the propagation direction of the $j$ beam from the form of Eq. (1) in the $(L)$ or $\left(L^{\prime}\right)$ frame to the form of Eq. (5) in the $(T)$ frame, where the diagonal incident angle $\xi$ of the beam is given by

$$
\begin{aligned}
& \cos \xi=\left(\cos ^{2} \beta \cos ^{2} \gamma+\sin ^{2} \beta\right)^{1 / 2}, \\
& \sin \xi=-\cos \beta \sin \gamma .
\end{aligned}
$$

After rotation from the $(L)$ or $\left(L^{\prime}\right)$ frame to the $(T)$ frame, an $e$-polarized or $m$-polarized incident beam becomes a mixture of $\epsilon$-polarized and $\mu$-polarized incident beams by means of

$$
\begin{aligned}
& {\left[\begin{array}{c}
\hat{E}_{j, L}^{e, \text { inc }} \\
\hat{E}_{j, L}^{m, \text { inc }}
\end{array}\right]=\left[\begin{array}{cc}
\cos \psi & \sin \psi \\
-\sin \psi & \cos \psi
\end{array}\right]\left[\begin{array}{l}
\hat{E}_{j, T}^{\epsilon, \text { inc }} \\
\hat{E}_{j, T}^{\mu, \text { inc }}
\end{array}\right],} \\
& {\left[\begin{array}{c}
\hat{B}_{j, L}^{e, \text { inc }} \\
\hat{B}_{j, L}^{m, \text { inc }}
\end{array}\right]=\left[\begin{array}{cc}
\cos \psi & \sin \psi \\
-\sin \psi & \cos \psi
\end{array}\right]\left[\begin{array}{l}
\hat{B}_{j, T}^{\epsilon, \text { inc }} \\
\hat{B}_{j, T}^{\mu, \text { inc }}
\end{array}\right],}
\end{aligned}
$$


where

$$
\begin{aligned}
& \cos \psi=\cos \gamma / \cos \xi, \\
& \sin \psi=\sin \beta \sin \gamma / \cos \xi .
\end{aligned}
$$

The center of the beam waist $\left(x_{0}, y_{0}, z_{0}\right)$ in the $(T)$ frame is given by

$$
\left[\begin{array}{l}
x_{0} \\
y_{0} \\
z_{0}
\end{array}\right]=\left[R_{j}\right]\left[\begin{array}{c}
-x_{\mathrm{fib}} \\
-y_{\mathrm{fib}} \\
0
\end{array}\right],
$$

and the position of a point on the receiver aperture is

$$
\left[\begin{array}{c}
x_{T}^{d} \\
y_{T}^{d} \\
z_{T}^{d}
\end{array}\right]=\left[R_{j}\right]\left[\begin{array}{c}
x_{L}^{d}-x_{\mathrm{fib}} \\
y_{L}^{d}-y_{\mathrm{fib}} \\
z_{L}^{d}
\end{array}\right] .
$$

The horizontal and vertical scattering angles $\theta$ and $\eta$ of a point on the receiver aperture are then given by

$$
\begin{aligned}
\cos \theta & =\frac{x_{T}^{d}}{\left[\left(x_{T}^{d}\right)^{2}+\left(y_{T}^{d}\right)^{2}\right]^{1 / 2}}, \\
\sin \theta & =\frac{y_{T}^{d}}{\left[\left(x_{T}^{d}\right)^{2}+\left(y_{T}^{d}\right)^{2}\right]^{1 / 2}}, \\
\tan \eta & =\frac{z_{T}^{d}-z_{0}}{\left\{\left[\left(x_{T}^{d}\right)^{2}+\left(y_{T}^{d}\right)^{2}\right]^{1 / 2}-x_{0}\right\}},
\end{aligned}
$$

with

$$
\rho_{T}=\rho_{L^{\prime}} .
$$

\section{Computation of Scattering in the Theory Frame}

For scattering of a diagonally incident plane wave by a fiber whose symmetry axis coincides with the $z_{T}$ axis, the partial-wave scattering amplitudes for an $\epsilon$-polarized incident plane wave to produce $\epsilon$-polarized scattered waves are $a_{l}(h)$, where $l$ is the partial-wave number and $h$ is the cylindrical coordinate system continuous index that describes the expansion of an incident beam in an angular spectrum of plane waves. Similarly, the partial-wavescattering amplitudes for a $\mu$-polarized incident plane wave to produce $\mu$-polarized scattered waves are $b_{l}(h)$. The partial-wave-scattering amplitudes for cross-polarized scattering (i.e., an $\epsilon$-polarized incident plane wave produces $\mu$-polarized scattered waves, or a $\mu$-polarized incident plane wave produces $\epsilon$-polarized scattered waves) are $q_{l}(h)$. Crosspolarized scattering occurs only for a diagonally incident plane wave and is impossible for scattering by a homogeneous sphere. Expressions for $a_{l}(h), b_{l}(h)$, and $q_{l}(h)$ are given in Refs. 10 and 11. If an incident beam is transversely localized, as is the case for a focused Gaussian beam, the partial-wave beamshape coefficients $A_{l}(h)$ and $B_{l}(h)$, which describe the expansion scalar radiation potential of the incident beam in terms of the eigenfunctions of the scalar Helmholtz equation, must also be employed.

For a Gaussian beam whose electric-field half- width satisfies $w_{0} \gg \lambda$, the beam-shape coefficients are accurately approximated by the so-called localized approximation. Note that for our fiber applications, $w_{0}=100-300 \mu \mathrm{m}$ and $\lambda<1 \mu \mathrm{m}$, so that the above restriction is easily satisfied. For an $\epsilon$-polarized incident beam the localized approximation is ${ }^{10}$

$$
\begin{aligned}
A_{l}(h)= & \frac{\cos \xi}{\left[2 s\left(1-h^{2}\right)(\pi F)^{1 / 2}\right]} \\
& \times \exp \left\{-i k\left[h z_{0}+\left(1-h^{2}\right)^{1 / 2} x_{0}\right]\right\} \\
& \times \exp \left\{-\left[h \cos \xi+\left(1-h^{2}\right)^{1 / 2} \sin \xi\right]^{2} / 4 s^{2}\right\} \\
& \times \exp \left\{-s^{2}\left[k y_{0}+l\left(1-h^{2}\right)^{-1 / 2}\right]^{2} / F\right\}, \\
B_{l}(h)= & 0,
\end{aligned}
$$

where the beam confinement parameter is given by

$$
\begin{aligned}
s= & 1 / k w_{0}, \\
F= & \cos \xi\left[\cos \xi-h\left(1-h^{2}\right)^{-1 / 2} \sin \xi\right] \\
& -2 i s^{2} k x_{0} /\left(1-h^{2}\right)^{1 / 2} .
\end{aligned}
$$

For a $\mu$-polarized incidence beam in the localized approximation, the expressions for $A_{l}(h)$ and $B_{l}(h)$ are interchanged. The $s \rightarrow 0$ limit corresponds to plane-wave incidence.

After the plane-wave incident partial-wave scattering amplitudes are multiplied by the incident-beamshape coefficients as in Ref. 10, computation of scattering of a focused Gaussian beam by a fiber proceeds relatively straightforwardly. Specifically, the three partial-wave-summed scattering amplitudes $S_{\epsilon}, S_{\mu}$, and $S_{q}$ are evaluated at the horizontal and vertical scattering angles $\theta$ and $\eta$ of a point on the receiver aperture,

$$
\begin{aligned}
S_{\epsilon}(\theta, h)= & \sum_{l=-\infty}^{\infty} \exp (i l \theta) a_{l}(h) \\
& \times \exp \left\{-s^{2}\left[k y_{0}+l\left(1-h^{2}\right)^{-1 / 2}\right]^{2} / F\right\}, \\
S_{\mu}(\theta, h)= & \sum_{l=-\infty}^{\infty} \exp (i l \theta) b_{l}(h) \\
& \times \exp \left\{-s^{2}\left[k y_{0}+l\left(1-h^{2}\right)^{-1 / 2}\right]^{2} / F\right\}, \\
S_{q}(\theta, h)= & \sum_{l=-\infty}^{\infty} \exp (i l \theta) q_{l}(h) \\
& \times \exp \left\{-s^{2}\left[k y_{0}+l\left(1-h^{2}\right)^{-1 / 2}\right]^{2} / F\right\} .
\end{aligned}
$$

The far-zone association of the continuous index $h$ with the propagation directions of the plane waves in the angular spectrum of the incident beam,

$$
\begin{aligned}
& \cos \eta=\left(1-h^{2}\right)^{1 / 2}, \\
& \sin \eta=h,
\end{aligned}
$$

is also employed. Equation (31) then becomes

$$
F=\left[\cos \xi \cos (\xi+\eta)-2 i s^{2} k x_{0}\right] / \cos \eta,
$$


and the amplitude and phase factors of the scattered wave in Eq. (38) are

$$
\begin{aligned}
\epsilon_{0}= & F^{-1 / 2} \exp \left[-\sin ^{2}(\xi+\eta) / 4 s^{2}\right] \exp \left[i k \left(\rho_{T}-x_{0} \cos \eta\right.\right. \\
& \left.\left.-z_{0} \sin \eta\right)\right] .
\end{aligned}
$$

If the incident $\mathrm{P} / \mathrm{D}$ beams are $e$ polarized, the $j$-scattered electric field at a point on the receiver aperture in the $(T)$ frame is then ${ }^{10}$

$$
\begin{aligned}
\mathbf{E}_{j, T}^{\text {scatt }}= & \epsilon_{0} \hat{\mathbf{E}}_{j, T}^{\epsilon, \text { scatt }}\left(S_{\epsilon} \cos \psi+S_{q} \sin \psi\right) \\
& +\epsilon_{0} \hat{\mathbf{E}}_{j, T}^{\mu, \text { scatt }}\left(-S_{q} \cos \psi+S_{\mu} \sin \psi\right) .
\end{aligned}
$$

The expressions for the scattered magnetic field, and for the scattered fields when the incident $\mathrm{P} / \mathrm{D}$ beams are $m$ polarized, are similar.

\section{Transformation of the Scattered Fields from the Theory Frame to the Translated Laboratory Frame}

The unit vectors of the $\epsilon$-polarized and $\mu$-polarized scattered electric and magnetic fields in the $\theta, \eta$ direction, corresponding to a point on the receiver aperture in the $(T)$ frame, are again a mixture of $e$-polarized and $m$-polarized scattered fields in the $\left(L^{\prime}\right)$ frame. But since the rotation angle of the mixture is quite complicated, we instead choose to apply the inverse rotation matrix $\left[\mathrm{R}_{j}\right]^{-1}$ to the $\epsilon-$ and $\mu$-polarization unit vectors to determine their $x_{L}, y_{L}$, and $z_{L}$ components in the $\left(L^{\prime}\right)$ frame directly. Specifically, we have

$$
\hat{\mathbf{E}}_{j, L^{\prime}}^{\epsilon, \text { scatt }}=\left[R_{j}\right]^{-1} \hat{\mathbf{E}}_{j, T}^{\epsilon, \text { scatt }},
$$

with similar expressions for the $\epsilon$-polarized scattered magnetic field unit vector and the $\mu$-polarized scattered electric and magnetic field unit vectors in the $\theta$, $\eta$ direction in the $(T)$ frame. These expressions are then combined with Eq. (39) to obtain the components of the $j$-scattered fields, $\mathbf{E}_{j, L^{\prime}}^{\text {scatt }}$ and $\mathbf{B}_{j, L^{\prime}}^{\text {scatt }}$, in the $\left(L^{\prime}\right)$ frame.

\section{Superposition of the Scattered Fields and Construction of the Total Poynting Vector}

Once the $x_{L}, y_{L}$, and $z_{L}$ components of both the $j=1$ and the $j=2$ scattered fields have been obtained for a point on the receiver aperture, the individual field components are added in the $\left(L^{\prime}\right)$ reference frame according to

$$
\begin{aligned}
& \mathbf{E}_{L^{\prime}}^{\text {total }}=\mathbf{E}_{1, L^{\prime}}^{\text {scatt }}+\mathbf{E}_{2, L^{\prime}}^{\text {scatt }}, \\
& \mathbf{B}_{L^{\prime}}^{\text {total }}=\mathbf{B}_{1, L^{\prime}}^{\text {scatt }}+\mathbf{B}_{2, L^{\prime}}^{\text {scat }} .
\end{aligned}
$$

for either $e$-polarized or $m$-polarized incident beams. The total scattered Poynting vector $S^{\text {total }}$ is then constructed according to

$$
\begin{aligned}
S^{\text {total }}= & \operatorname{Re}\left[\cos \chi^{\prime} \cos v^{\prime}\left(E_{y, L^{\prime}}^{\text {total* }} B_{z L^{\prime}}^{\text {total }}-E_{z L^{\prime}}^{\text {total }{ }^{\prime}} B_{y, L^{\prime}}^{\text {total }}\right)\right. \\
& +\cos \chi^{\prime} \sin v^{\prime}\left(E_{z, L^{\prime}}^{\text {total }} B_{x, L^{\prime}}^{\text {total }}-E_{x, L^{\prime}}^{\text {total }} B_{z, L^{\prime}}^{\text {total }}\right) \\
& \left.+\sin \chi^{\prime}\left(E_{x, L^{\prime}}^{\text {total* }} B_{y, L^{\prime}}^{\text {total }}-E_{y, L^{\prime}}^{\text {total* }} B_{x, L^{\prime}}^{\text {total }}\right)\right] .
\end{aligned}
$$

Finally, the total scattered Poynting vector is integrated over the receiver aperture to produce the integrated detector power.

\section{B. Modeling the Receiver Assembly}

In addition to fiber tilt and finite-beam-size effects, there are additional factors that have not been included in the $\mathrm{P} / \mathrm{D}$ model that could affect the computed phase-shift distribution. Among these factors is the propagation of light through the receiver assembly to the optical detectors. For the commercial TSI system considered in these studies, the relevant receiver components consist of two achromats, two plane mirrors, and a rectangular slit aperture. The two mirrors are used to produce a folding optical cavity that results in a more compact receiver assembly. As a fiber is moved within the crossed-beam measurement volume, the slit aperture serves to limit the extent of the measurement volume over which light can be collected. In the commercial TSI receivers, the two achromats are each split in half and mounted in separate receiver assemblies. Therefore, the entrance aperture of each receiver consists of a semicircular collection area. Modeling of the receiver assemblies is important, because it provides the ability to account for changing effective receiver sizes and angles (i.e., signal clipping) and can be used in modeling the effective size of the measurement volume.

To model the receiver assembly of the system, we took a straightforward geometrical-optics ray-trace approach with the simple objective of determining which scattered light rays incident on the entrance aperture of the receivers will pass through the slit aperture and therefore contribute to the measured signal intensity. The general approach consists of determining the propagation direction of the light emerging from the fiber given an integration point on the receiver lens and the fiber location. Given knowledge regarding the surface curvatures and separations of the lens surfaces, one can determine the path of the light ray, using Snell's law. Once the ray reaches the plane of the slit aperture, the coordinates of the ray intersection point are compared with the physical dimensions of the slit aperture to determine if the ray will pass through the aperture. In this paper we do not present the mathematical development for the ray-trace analysis but instead refer the reader to a previously published study ${ }^{12}$ in this area.

Note that this ray-trace approach is used only to determine if a scattered ray emerging from the fiber (assumed a point source) will pass through the slit aperture. As such, it should be considered a first check to determine if light incident at a given integration point on the receiver entrance apertures will contribute to the total integrated power collected by the receiver. Even if the above analysis concludes that the geometric ray does pass through the aperture, the actual intensity in such a ray could be zero (or extremely small), owing to the relationship between the integration point and particular tilt or location of the fiber in the measurement volume. 


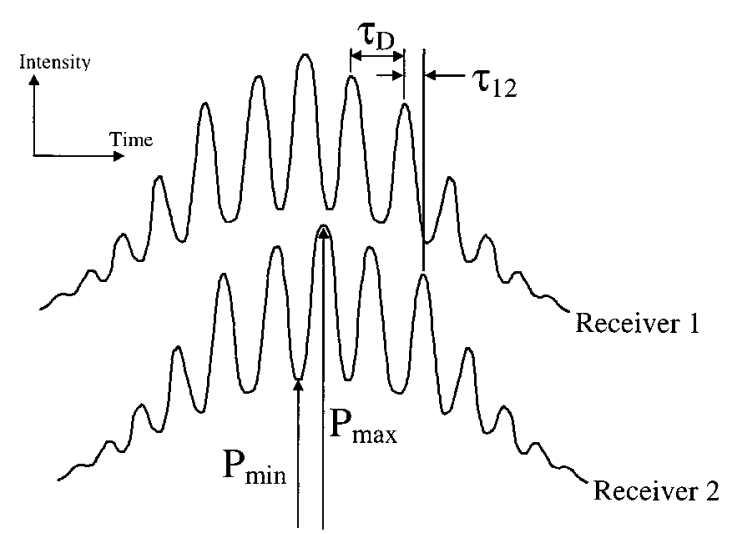

Fig. 2. Typical Doppler bursts illustrating the measurable quantities of interest.

\section{Phase/Doppler Output Quantities of Interest}

For the generalized $\mathrm{P} / \mathrm{D}$ computer model we evaluate the output quantities (i.e., phase shift, visibility, and amplitude) in a similar manner as was presented in an earlier study. ${ }^{6}$ Below, we briefly summarize the approach.

If one calculates the integrated detector power as a function of beam phase shift, a sinusoidally varying function is observed. For the tilted fiber calculation, however, the integration is no longer one dimensional but must now be performed over the entire area of the receiver entrance aperture,

$$
P_{n}=\iint S_{\text {aperture }} S^{\text {total }} \mathrm{d} x_{\text {rel }} \mathrm{d} y_{\text {rel }}
$$

where the subscript $n$ denotes the receiver under consideration. For detectors at two different angular locations ( $n=1,2)$ one obtains two similar functions. However, they are, in general, different in terms of amplitude, zero offset, and relative phase. An example showing typical $\mathrm{P} / \mathrm{D}$ output signals is shown in Fig. 2. The high-frequency portion of the signal (with period $\tau_{D}$ ) results from the fiber traversing the fringe planes and/or by movement of the fringes past the fiber, because of the beam frequency shift introduced by the Bragg cell. The Gaussian envelope of the signal is due to the Gaussian intensity variation across each focused laser beam. Note that for a fiber positioned statically in the measurement volume, only the high-frequency variation can be observed, and it is this portion of the signal that carries the diameter information. In general, the output from the detector can be written in the form

$$
P_{n}=A_{n}+B_{n} \sin \left(\varphi_{B}-\varepsilon_{n}\right),
$$

where $\varphi_{B}$ is the beam 2 phase shift (from the Bragg cell), $\varepsilon_{n}$ is the phase offset, and $A_{n}$ and $B_{n}$ are constants. Note that in this expression we consider only the high-frequency component of the signal. From this general form of the detector response, one can determine several quantities directly relevant to the $\mathrm{P} / \mathrm{D}$ measurement method. The first of these is the signal visibility $V$, which is defined as

$V_{n}=\frac{P_{\max }-P_{\min }}{P_{\max }+P_{\min }}=\frac{\left(A_{n}+B_{n}\right)-\left(A_{n}-B_{n}\right)}{\left(A_{n}+B_{n}\right)+\left(A_{n}-B_{n}\right)}=\frac{B_{n}}{A_{n}}$.

The detector phase shift can be determined from

$$
\tau_{12}=\left|\varepsilon_{2}-\varepsilon_{1}\right| .
$$

Following the development of Ref. 6, the detector phase offset is determined with

$\tan \varepsilon_{n}=\left[\frac{\left(\sin \varphi_{1}-\sin \varphi_{3}\right)-\tilde{P}_{n}\left(\sin \varphi_{2}-\sin \varphi_{3}\right)}{\tilde{P}_{n}\left(\cos \varphi_{3}-\cos \varphi_{2}\right)-\left(\cos \varphi_{3}-\cos \varphi_{1}\right)}\right]$,

where

$$
\tilde{P}_{n}=\frac{P_{n}\left(\varphi_{1}\right)-P_{n}\left(\varphi_{3}\right)}{P_{n}\left(\varphi_{2}\right)-P_{n}\left(\varphi_{3}\right)} .
$$

The signal amplitude ( $B$ coefficient) can then be determined with

$$
B_{n}=\frac{P_{n}\left(\varphi_{2}\right)-P_{n}\left(\varphi_{3}\right)}{\sin \left(\varphi_{2}-\varepsilon_{n}\right)-\sin \left(\varphi_{3}-\varepsilon_{n}\right)},
$$

and the dc component ( $A$ coefficient) from

$$
A_{n}=P_{n}\left(\varphi_{3}\right)-B_{n} \sin \left(\varphi_{3}-\varepsilon_{n}\right) .
$$

\section{Analytical Results and Computational Checks}

\section{A. Analytical Results}

When a Gaussian beam is diagonally incident on a long dielectric fiber, the scattered wave in the far zone is a thin conical shell. The axis of the conical shell coincides with the fiber symmetry axis, and the cone's apex is the point where the beam crosses the fiber axis. The cone's opening half-angle is $\pi / 2-\xi$, and the shell includes the undeflected beam in the $\eta$ $=(-\xi)$ direction. Since the scattered fields are proportional to $\exp \left[-\sin ^{2}(\xi+\eta) / 4 s^{2}\right]$ as in Eq. (38), the shell has the $1 / e$ angular half-thickness

$$
\Delta \eta=2 s,
$$

if $\xi$ and $\eta$ are small. For $\lambda=0.5145 \mu \mathrm{m}$ and $w_{0}=50 \mu \mathrm{m}$, for example, the angular $1 / e$ halfthickness of the conical shell is $\Delta \eta=0.19^{\circ}$, which corresponds to $1.66 \mathrm{~mm}$ on a detector plane $\rho_{L}=500$ $\mathrm{mm}$ away. The light intensity within the conical shell in the $\theta$ direction is proportional to the square of the magnitudes of the partial-wave-summed scattering amplitudes.

When the two $\mathrm{P} / \mathrm{D}$ beams are incident on the fiber, the two scattered conical shells of light overlap to a greater or lesser extent, depending on both the fiber's tilt angles and its displacement from the origin of the $(L)$ frame. If the fiber lies along the $z_{L}$ axis and the beams are normally incident with $\xi=0^{\circ}$, the scattered conical shells become planes and completely overlap. Since the $\mathrm{P} / \mathrm{D}$ signal is 
contained in the interference of the scattered electric fields of the two beams, the case of perfect overlap gives a high-visibility $\mathrm{P} / \mathrm{D}$ interference signal. However, if the fiber is tilted so that the two scattered conical shells do not overlap at the detector, no $\mathrm{P} / \mathrm{D}$ interference signal is produced. Thus the visibility of the $\mathrm{P} / \mathrm{D}$ interference signal sensitively depends on the fiber's tilt angles $\gamma$ and $\delta$. If the two conical shells of scattered light are shifted with respect to each other along the common cone axis, owing to a displacement of the fiber from the origin of the $(L)$ frame, the visibility of the P/D interference signal also decreases.

To estimate the dependence of the $\mathrm{P} / \mathrm{D}$ signal on both the tilt and displacement of the fiber in the $(L)$ frame, we consider beams with $\lambda=0.5145 \mu \mathrm{m}$ and $w_{0}=50 \mu \mathrm{m}$, as above, and a beam crossover angle of $\alpha=5^{\circ}$. First, when the fiber is tilted in the $\delta=0^{\circ}$ and $180^{\circ}$ directions (i.e., in-beam tilt) for arbitrary $\gamma$ and $x_{\text {fib }}=y_{\text {fib }}=0$, Eqs. (16) and (17) show that $\xi_{1}=\xi_{2}$, and thus the two scattered conical shells of light completely overlap, thus giving no decrease in the visibility of the $\mathrm{P} / \mathrm{D}$ interference signal. The only effect is a shift in the location of the scattered light at the detector. But when $\delta=90^{\circ}$ and $270^{\circ}$ (i.e., cross-beam tilt), one scattered conical shell opens above the $x_{T}-y_{T}$ plane of the $(T)$ frame (i.e., $\xi_{1}>0^{\circ}$ ), whereas the other opens below the plane (i.e., $\xi_{2}<0^{\circ}$ ). If $\gamma$ is small, the $1 / e^{2}$ intensity point of the first conical shell coincides with the center point of the second when

$$
\gamma=(2)^{1 / 2} s / \alpha \approx 1.52^{\circ}
$$

for the parameters given above. If the fiber is tilted greater than this amount when $\delta=90^{\circ}$ and $270^{\circ}$, the visibility of the $\mathrm{P} / \mathrm{D}$ signal should dramatically decrease.

Second, when the fiber has no tilt in the $(L)$ frame but crosses the $x_{L}-y_{L}$ plane at $\left(x_{\text {fib }}, y_{\text {fib }}, 0\right)$, the center of the focal waist of each of the two incident beams in the $(T)$ frame has different $\left(x_{0}, y_{0}, z_{0}\right)$ coordinates as in Eq. (22). This causes the incident beams to cross the fiber at different positions along its axis. Since the apex of each conical shell of scattered light is the point where the incident beam crosses the fiber axis, a displacement of the fiber in the $(L)$ frame produces different vertical shifts of the scattered conical shells along the common cone axis, and the overlap of the conical shells again decreases. The vertical shift of each beam in the $(T)$ frame is

$$
\begin{aligned}
\Delta z_{T} & =z_{0}+x_{0} \tan \xi \\
& =-\gamma y_{\text {fib }} \sin \delta \pm \gamma \alpha\left(x_{\text {fib }} \sin \delta+y_{\text {fib }} \cos \delta\right) .
\end{aligned}
$$

Again, if $\gamma$ is small, the $1 / e^{2}$ intensity point of the first conical shell coincides with the center point of the second when

$$
\gamma=\left[(2)^{1 / 2} s / \alpha\right]\left(\rho_{T} / \rho_{\mathrm{fib}}\right),
$$

where

$$
\rho_{\mathrm{fib}}=\left[\left(x_{\mathrm{fib}}\right)^{2}+\left(y_{\mathrm{fib}}\right)^{2}\right]^{1 / 2} .
$$

Since $\rho_{T} / \rho_{\text {fib }} \gg 1$, the visibility of the $\mathrm{P} / \mathrm{D}$ signal should depend on the fiber's degree of tilt more sensitively than it does on its displacement from the origin of the $(L)$ frame.

\section{B. Code Verification and Testing}

The GPDCM development was implemented as a FORTRAN computer code. Several tests of the code were conducted to verify the accuracy of the model. First, during the subroutine development stage, tests were conducted on the subroutines employed by the tilted beam computer model. The subroutines were extensively tested for the correctness of the method of computation of the Bessel functions and Neumann functions; the plane-wave partial-wave scattering amplitudes $a_{l}, b_{l}$, and $q_{l}$; the recursion formula for the angular functions; the summed scattering amplitudes $S_{\epsilon}, S_{\mu}$, and $S_{q}$; and the handling of the interior and exterior fiber size parameters for the diagonal incidence geometry. The scattered intensity for a single incident Gaussian beam was then computed for the range of beam widths $\left(w_{0} \gg a\right)$ to $\left(w_{0} \ll a\right)$. It was numerically verified that for $w_{0} \gg a$ the scattered intensity was indistinguishable from that of plane-wave scattering. As $w_{0} \approx a$, it was verified that edge-ray phenomena such as rainbows and large-angle transmission are damped, owing to smaller input light intensity at the edges of the fiber. For $w_{0} \ll a$ it was verified that all side scattering was lost and that only forward scattering and backscattering remain.

The performance of the integrated code that incorporates all aspects of the GPDCM was also verified with several additional tests. The computer model was first used to compute the results for the limiting case that corresponds to a perpendicular fiber $(\gamma=0)$ illuminated by incident beams that possess very large beam waists, i.e., $w_{0}=10^{4} \mu \mathrm{m}$. Under these conditions the GPDCM provided results equivalent to the plane-wave $\mathrm{P} / \mathrm{D}$ model developed in an earlier study. ${ }^{6}$ We also tested the computer model by verifying that the computed results were independent of the azimuthal angle $\delta$, when $\gamma=0^{\circ}, 180^{\circ}$, and we performed test runs, comparing the dependence of the scattered light distribution at the receiver for a tilted fiber with the analytical predictions of Subsection 3.A. The results of calculations are shown in Fig. 3. These results have been generated by integration of the power over the beam phase shift $\varphi_{B}$. For a perpendicular fiber (i.e., $\gamma=0^{\circ}$, arbitrary $\delta$ ), the computed scattered-light pattern is a thin horizontal sheet in the $z_{L}=0$ plane. As the fiber is tilted in-beam, the computed light pattern moved vertically on the face of the receiver but the scattered light from the two beams still overlapped. For cross-beam fiber tilt the computed light pattern also moved vertically, but in addition the two scattered light cones began to separate at the receivers. In both cases 


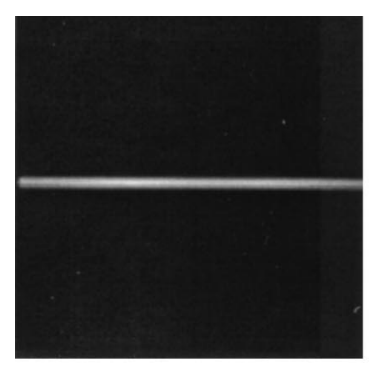

$$
\gamma=0^{\circ}, \delta=90^{\circ}
$$

No Tilt
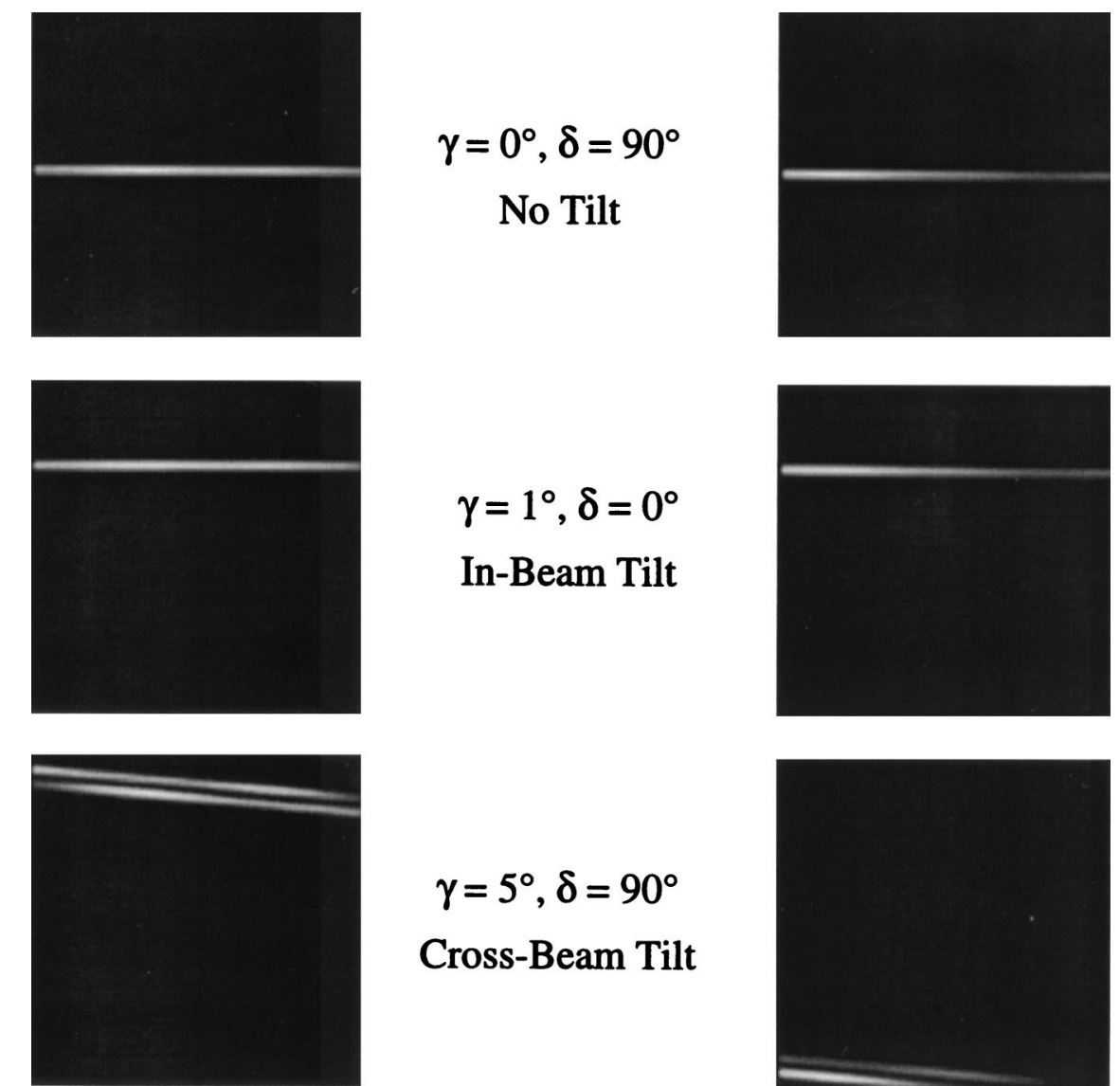

$\gamma=5^{\circ}, \delta=90^{\circ}$

Cross-Beam Tilt
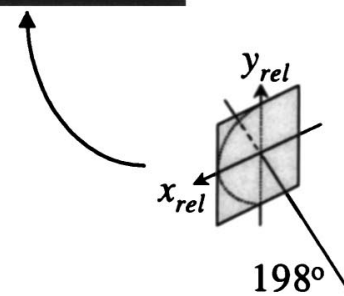

$-17.5 \mathrm{~mm}<x_{\text {rel }}<17.5 \mathrm{~mm}$ $-17.5 \mathrm{~mm}<y_{\text {rel }}<17.5 \mathrm{~mm}$

Fig. 3. Computed scattered-light distribution for an $18.3-\mu \mathrm{m}$ diameter fiber: $\lambda=514.5 \mathrm{~nm}, 1.52$ refractive index, $500 \mathrm{~mm}$ focal lengths, 17 -mm beam separation, $w_{0}=150 \mu \mathrm{m}$, parallel $e$-field polarization.

these computed light patterns are consistent with both experimental observation and the analytical predictions of Subsection 3.A.

\section{Computation Results}

In this section we outline several general observations regarding issues that are important in the design of a P/D system for measurement of cylindrical fibers. In support of these observations and recommendations, we present a limited set of supporting computations that were generated with the GPDCM.
A. Comparison between Backscatter and Sidescatter Arrangements

Earlier research ${ }^{6}$ demonstrated that several P/D configurations can potentially be used for fiber-diameter measurements. Among these configurations, two are particularly notable. The sidescatter arrangement, in which the receivers are positioned nominally perpendicular to the incident beams, provides the best phase-diameter linearity, as defined by Eq. (22) of Ref. 6. The primary drawback of the sidescatter arrangement is the relatively large footprint of the 

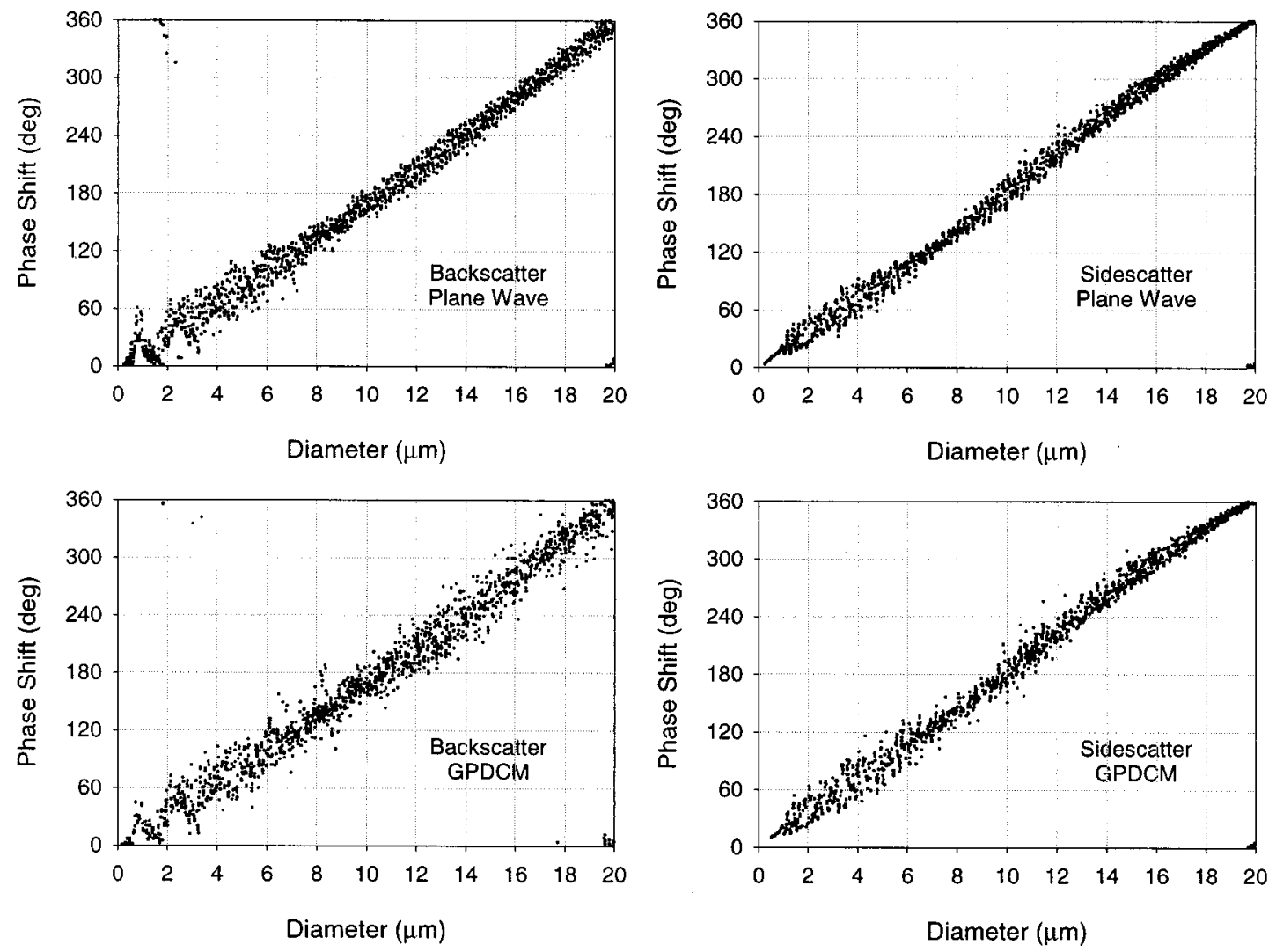

Fig. 4. Phase shift as a function of diameter computed with the plane wave and the GPDCM for both backscatter and sidescatter P/D arrangements.

instrument, which results in added alignment and stability difficulties for actual applications. Alternatively, the backscatter arrangement, in which the receivers are positioned nominally $\pm 20^{\circ}$ from backscatter, provides a much smaller instrument footprint, thus resulting in a more compact and stable system. However, the phase-diameter response exhibited by the backscatter arrangement is not as linear as the sidescatter arrangement, thus resulting in larger diameter measurement uncertainties. We note that this earlier study ${ }^{6}$ was conducted with the plane-wave, perpendicular fiber theoretical model, which did not account for fiber tilt, translation, and finite aperture effects. To understand the influence of these additional factors, we performed additional simulations using the GPDCM developed in the preceding sections.

Since the GPDCM includes the effects of finite beam size, the computed results show significant variations in terms of relative intensity and visibility of the scattered light signals as fiber tilt angles and the position of the fiber are varied within the measurement volume. To examine the output from the GPDCM in a meaningful manner, we must determine approximate thresholds on both signal intensity and visibility that will define a validated measurement. To determine these approximate thresholds for intensity and visibility, we examined the output from the GPDCM for the special case that corresponds to a perpendicular, nominal $15-\mu$ m-diameter fiber, positioned at the center of the $\mathrm{P} / \mathrm{D}$ measurement volume. Assuming that a 14.9-15.1- $\mu \mathrm{m}$-diameter range represents an approximate upper limit on diameter for our application of interest, the average intensities would correspond to an approximate upper limit on the magnitude of the scattered-light signal we would expect to measure at each receiver. Assuming that the hardware is configured such that these computed intensities correspond to approximate detector saturation, we can then determine an estimation of the minimum threshold intensity by dividing the $\sim 15$ $\mu \mathrm{m}$-diameter average saturation intensity by the dynamic range of the detectors. For our studies we considered the case that corresponds to a detector dynamic range of 1000 . For visibility we assumed minimum threshold values that correspond to $1 \%$ of the maximum calculated values. Note that the signal intensity thresholds differ for the backscatter and sidescatter arrangements.

Figure 4 presents the calculated phase shifts as a function of fiber diameter, computed with both the plane-wave, perpendicular fiber model and the GPDCM, for both the backscatter and sidescatter $\mathrm{P} / \mathrm{D}$ configurations shown in Table 1 . To generate Fig. 4, the tilt angles and the position of the fiber, as well as the diameter, were chosen randomly within the approximate detection limits given in Table 1 . The scattering angles used in the $\mathrm{P} / \mathrm{D}$ arrange- 


\begin{tabular}{|c|c|c|}
\hline Input Quantity & Backscatter & Sidescatter \\
\hline Refractive index & 1.52 & 1.52 \\
\hline Incident beam wavelength & $514.5 \mathrm{~nm}$ & $514.5 \mathrm{~nm}$ \\
\hline Receiver angles & $160^{\circ}$ and $196^{\circ}$ & $82^{\circ}$ and $278^{\circ}$ \\
\hline Transmitter/receiver focal lengths & $500 \mathrm{~mm}$ & $500 \mathrm{~mm}$ \\
\hline Radius of semicircular receivers & $35 \mathrm{~mm}$ & $35 \mathrm{~mm}$ \\
\hline Beam separation & $17 \mathrm{~mm}$ & $13 \mathrm{~mm}$ \\
\hline Electric field polarization & Along fiber axis ( $m$ pol) & Perp. to fiber axis (e pol) \\
\hline Gaussian beam waist radius & $150 \mu \mathrm{m}$ & $150 \mu \mathrm{m}$ \\
\hline Slit aperture dimensions & $200 \mu \mathrm{m} \times 400 \mu \mathrm{m}$ & $200 \mu \mathrm{m} \times 400 \mu \mathrm{m}$ \\
\hline Range of tilt angle $\gamma$ & $0^{\circ}<\gamma<7^{\circ}$ & $0^{\circ}<\gamma<7^{\circ}$ \\
\hline Range of tilt angle $\delta$ & $0^{\circ}<\delta<360^{\circ}$ & $0^{\circ}<\delta<360^{\circ}$ \\
\hline Range of fiber position $x_{L}$ & $-1020 \mu \mathrm{m}<x_{L}<1020 \mu \mathrm{m}$ & $-330 \mu \mathrm{m}<x_{L}<330 \mu \mathrm{m}$ \\
\hline Range of fiber position $y_{L}$ & $-270 \mu \mathrm{m}<y_{L}<270 \mu \mathrm{m}$ & $-270 \mu \mathrm{m}<y_{L}<270 \mu \mathrm{m}$ \\
\hline
\end{tabular}

ments considered in this paper are similar to the arrangement examined in Ref. 6. In this study, however, we used experimental parameters (i.e., incident wavelength, optics specifications) that correspond to commercially available components. A comparison of the results in Fig. 4 shows significantly less scatter (i.e., better linearity) for the sidescatter arrangement. The sidescatter arrangement is also less influenced by the occurrence of tilted/translated fibers as evidenced by the relatively close agreement between the plane-wave and GPDCM results. Figure 4 also shows that tilt/ translation/aperture effects have a more significant impact on the larger-diameter fibers than on smaller fibers for the backscatter configuration, which is consistent with observations made during laboratory studies.

These results suggest that, when possible, the $\mathrm{P} / \mathrm{D}$ system should be used in the sidescatter arrangement for performing fiber diameter measurements. However, for applications such as on-line fiberglass measurements, backscatter $\mathrm{P} / \mathrm{D}$ arrangements provide several important practical advantages that must be considered when the overall optimum $\mathrm{P} / \mathrm{D}$ arrangement is evaluated.

\section{B. Impact of Receiver Shape}

The standard receiver assemblies in the commercial TSI P/D system makes use of two semicircular collection lenses that can be positioned at arbitrary locations in space. Although this geometry proves to be useful in spherical aerosol sizing, calculations we performed suggest that this may not be the optimum receiver geometry for fiber sizing. Both simulations and experiment have shown that when the fiber is tilted, the scattered light is, in general, displaced vertically at the receiver apertures, thus resulting in a change in the effective aperture size (Fig. 5).

To demonstrate the effect of the receiver aperture shape, we examined the computed phase response of the $\mathrm{P} / \mathrm{D}$ system for fibers in the $14.5-15.5-\mu \mathrm{m}-$ diameter range using the plane-wave model, GPDCM with a full semicircular receiver, and the GPDCM with a reduced vertical receiver size. The reduced vertical dimension could be easily incorporated into an actual $\mathrm{P} / \mathrm{D}$ system by placement of masks $\mathrm{M}_{1}$ and $\mathrm{M}_{2}$ at the entrance aperture to the receivers (Fig. 5). In particular, we are interested in how the standard deviation in the computed phase shifts (i.e., phase broadening) is influenced by the three configurations examined.

The results of the simulations for both backscatter and sidescatter $\mathrm{P} / \mathrm{D}$ arrangements are shown in Fig. 6 and illustrate several important points. As expected, the least amount of phase distribution broadening (i.e., smallest standard deviation in computed phase shift) is observed for the plane-wave models shown in Figs. 6(a) and 6(b). Broadening for the plane-wave cases is caused by high-frequency oscillations in the $\mathrm{P} / \mathrm{D}$ response curve but does not include the effects of fiber tilt/translation. For the backscatter configuration, shown in Fig. 6(c), a significant increase in standard deviation is observed, which results from the inclusion of the fiber tilt/translation and finite aperture effects. This phase broadening, however, can be reduced from approximately $17.9^{\circ}$ to $14.1^{\circ}$ [Fig. 6(e)] by reduction of the vertical extent of the receiver apertures from \pm 35 to $\pm 24 \mathrm{~mm}$. The narrower diameter distribution exhibited by [Fig. 6(e)] results from limiting the vertical extent of the receivers, which lessens the impact of

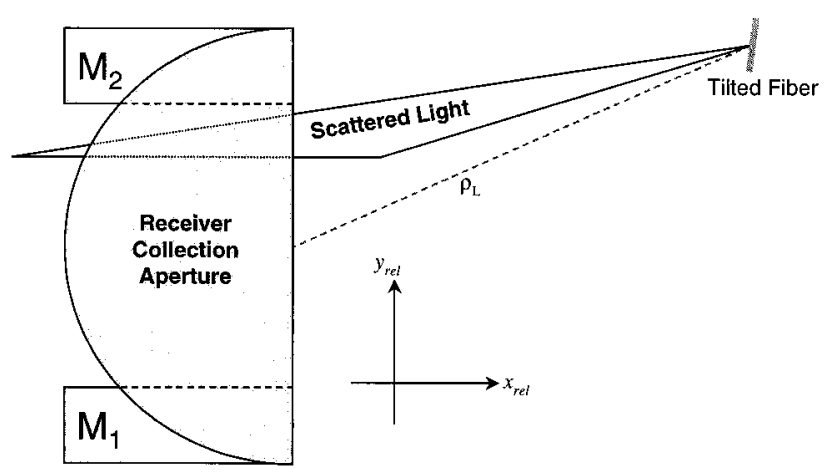

Fig. 5. Schematic illustrating the semicircular receiver aperture and the reduced aperture generated by application of masks $\mathrm{M}_{1}$ and $\mathrm{M}_{2}$. 

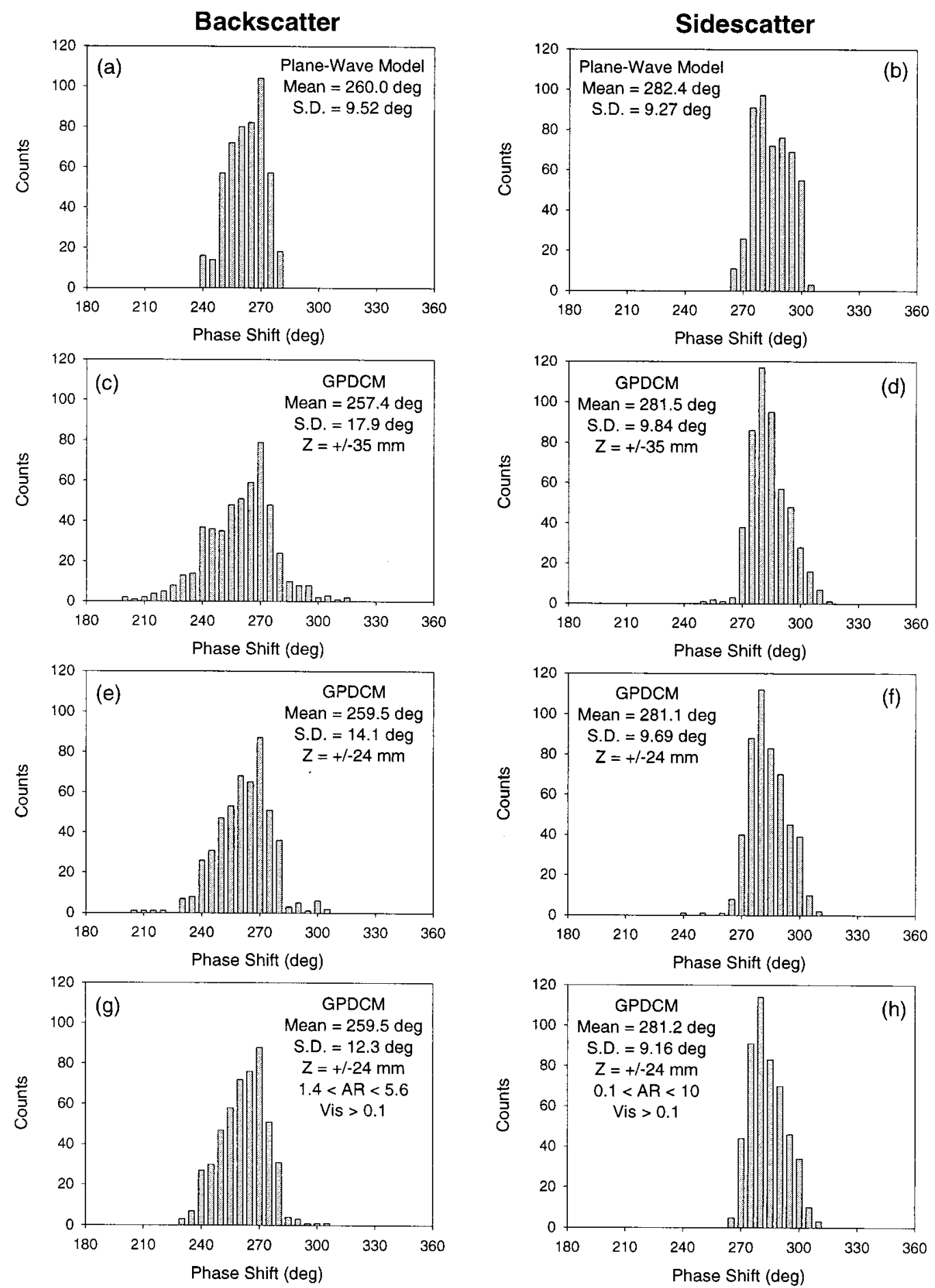

Fig. 6. Simulated phase distributions for fibers in the 14.5-15.5- $\mu \mathrm{m}$-diameter range. The $\mathrm{P} / \mathrm{D}$ parameters are given in Table 1; the total counts for all distributions are 500 .

in-beam fiber tilt by elimination of portions of the receiver where the largest changes in effective aperture size will occur. In contrast to the backscatter arrangement, the sidescatter arrangement is less influenced by the presence of tilted/translated fibers, with both the mean and standard deviations showing little variation between Figs. 6(b), 6(d), and 6(f). Interestingly, none of the factors considered in this study (i.e., tilt, translation aperture) has any significant impact on the value of the mean phase shifts that remain nearly constant for a given configuration, deviating by no more than $\sim 1 \%$ for either the backscatter or sidescatter arrangement. This result is consistent with results of experimental studies that suggest that mean diameter changes can be accurately tracked despite the inability to determine 
fiber distribution accurately, owing to phase broadening.

\section{Effect of Visibility and Amplitude Thresholds on Phase Distribution}

In addition to hardware considerations such as aperture shape, a different approach in dealing with fiber tilt is to attempt to eliminate measurements of tilted fibers during the signal-processing stage of the measurement. To accomplish this, one must be capable of identifying measurable signal characteristics that may be indicative of a tilted fiber that will produce an erroneous phase measurement. In this section we reexamine cases (e) and (f) of Fig. 6 to determine if reduced phase broadening would result if validations were limited only to those signals that satisfy certain criteria. Our approach consisted of evaluating the standard deviation of the computed phase shift for randomly chosen signal-processing values [i.e., minimum signal visibility threshold and acceptable range on amplitude ratio (AR)]. The only other requirement was that at least $50 \%$ of the signals must satisfy these criteria. The phase distributions that result from imposing these threshold criteria on signal characteristics are presented in Figs. 6(g) and 6(h). Note that the standard deviation of the phase shift with the backscatter configuration decreased from $14.1^{\circ}$ to $12.2^{\circ}$, whereas the standard deviation of the phase distribution with the sidescatter arrangement was also slightly reduced. As before, the mean phase shift remains essentially unchanged by the signal visibility and amplitude ratio threshold requirements. We note that by imposing these thresholds, the percentage of signals considered valid must correspondingly decrease. These results suggest that we can reduce the standard deviation of the computed phase shift at the expense of validation rates by imposing threshold criteria on signal amplitude ratio and/or visibility. We note, however, that implementation of such thresholding methods into actual signal-processing instrumentation is not a trivial matter.

\section{Conclusions}

We have presented a generalized phase/Doppler (P/D) computer (GPDCM) model for the response of the $\mathrm{P} / \mathrm{D}$ measurement system to laser light scattering by cylindrical fibers. We expect that this model will provide a valuable computational tool by which design and evaluation of $\mathrm{P} / \mathrm{D}$ fiber sizing systems can be performed. The GPDCM is valid for arbitrary fiber diameters and refractive indices, Gaussian incident beams, and it accounts for arbitrary fiber orientations, fiber positions, and effects that are due to the two-dimensional receivers. The calculations that we performed with both the plane wave and the GPDCM have provided important insights into the effects of fiber tilt, fiber translation, and finite slit aperture effects on the performance of the $\mathrm{P} / \mathrm{D}$ system. In addition, the results have provided guidance on system design modifications that may be useful in optimizing the performance of the
$\mathrm{P} / \mathrm{D}$ system for fiber measurement applications. Below, we summarize several of the important results and observations from the theoretical modeling efforts.

(1) In earlier research ${ }^{6}$ it was demonstrated that the linearity of the phase-diameter response is better for sidescatter experimental arrangements than for comparable backscatter arrangements. In the calculations presented in this paper, we have shown that fiber tilt and translation effects are also more pronounced in the backscatter configuration than in the sidescatter configuration. These results would suggest that, when possible, use of the P/D system for fiber diameter measurements should be performed in the sidescatter arrangement. However, for applications such as wool fiberglass measurements, backscatter $\mathrm{P} / \mathrm{D}$ arrangements provide several important advantages that must be considered when the optimum experimental arrangement is determined.

(2) For semicircular receiver entrance aperture, the observed phase broadening in the backscatter arrangement is dominated by in-beam fiber tilt that results in vertical displacement of the scattered light on the face of the receivers. This displacement results in reduction in the effective aperture size, which translates into variations in the phase measurements. Calculations have shown that use of rectangular collection apertures (or semicircular apertures with limited vertical extent) will reduce the standard deviation of the measured phase shifts.

(3) Despite the impact of fiber tilt/translation and finite aperture effects on measurement of the fiber distribution, these factors have only a minimal affect on the mean phase shift. From a practical viewpoint this means that the $\mathrm{P} / \mathrm{D}$ method can be used effectively for monitoring mean fiber diameter changes even though the ability to monitor distribution changes may be limited by fiber tilt/translation and finite aperture effects.

(4) Theoretical calculations have suggested that one can also reduce the standard deviation of the computed phase shift at the expense of signal validation rates by imposing threshold criteria on signal amplitude ratio and/or signal visibility.

The authors acknowledge the support of this research through the U.S. Department of Energy contract DE-FG03-96ER82229, project manager Rolf Butters.

\section{References}

1. M. Saffman, P. Buchhave, and H. Tanger, "Simultaneous measurement of size, concentration and velocity of spherical particles by a laser Doppler method," in Laser Anemometry in Fluid Mechanics II, R. J. Adrian, D. F. Durao, F. Durst, H. Mishina, and J. Whitelaw, eds. (LADOAN, Lisbon, Portugal, 1984), pp. 85-103.

2. S. V. Sankar and W. D. Bachalo, "Response characteristics of the phase Doppler particle analyzer for sizing particles larger than the light wavelength," Appl. Opt. 30, 1487-1496 (1991). 
3. A. Naqwi and F. Durst, "Analysis of laser light-scattering interferometric devices for in-line diagnostics of moving particles,” Appl. Opt. 32, 4003-4018 (1993).

4. S. V. Sankar, B. J. Weber, D. Y. Damemoto, and W. D. Bachalo, "Sizing fine particles with the phase Doppler interferometric technique," Appl. Opt. 30, 4914-4920 (1991).

5. S. A. Schaub, D. R. Alexander, and J. P. Barton, "Theoretical analysis of the effects of particle trajectory and particle resonances on the performance of a phase-Doppler particle analyzer," Appl. Opt. 33, 473-483 (1994).

6. S. A. Schaub, A. A. Naqwi, and F. L. Harding, "Design of a phase/Doppler light-scattering system for measurement of small-diameter glass fibers during fiberglass manufacturing," Appl. Opt. 37, 573-585 (1998).

7. H. Mignon, G. Grehan, G. Gouesbet, T. H. Xu, and C. Tropea, "Measurement of cylindrical particles with phase Doppler anemometry," Appl. Opt. 35, 5180-5190 (1996).
8. A. Naqwi and L. M. Jensen, "Device for interferometric measurements with compensation for tilt and position of measured cylindrical objects," U.S. Patent 5,513,004 (30 April 1996).

9. A. Naqwi, T. Mahon, D. Havir, P. Tsai, C. Hassenboehler, and L. Wadsworth, "On-line sizing of meltblown and spunbond fibers using adaptive phase/Doppler velocimeter (APV) method," in Book of Papers, INDA-TEC 95 (Association of Nonwoven Fabrics Industry, Cary, N.C., 1995), pp. 167-184.

10. J. A. Lock, "Scattering of a diagonally incident focused Gaussian beam by an infinitely long homogeneous circular cylinder," J. Opt. Soc. Am. A 14, 640-652 (1997).

11. C. F. Bohren and D. R. Huffman, Absorption and Scattering of Light by Small Particles (Wiley, New York, 1983).

12. J. B. Keller and H. B. Keller, "Determination of reflected and transmitted fields by geometrical optics," J. Opt. Soc. Am. 40, 48-52 (1950). 